\title{
Analysis of drug resistance of extended-spectrum beta- lactamases-producing Escherichia coli and Klebsiella pneumoniae in children with urinary tract infection
}

Lu Keshi, MD, Xiao Weiwei, MD, Li Shoulin, PhD, MD, Liu Xiaodong, MD Wang Hao, PhD, MD, Jiang Junhai, MD,

Wang Xiangwei, PhD, MD, Wang Rui, MD, Zhu Pei, MD.

\section{ABSTRACT}

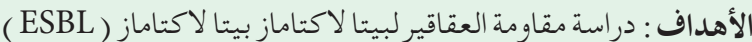

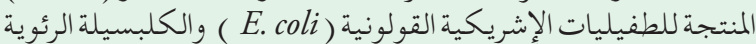
(

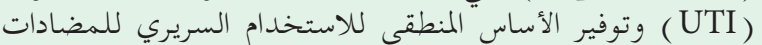
الحيوية.

المنهجية : أجري تحليل بأثر رجعي لمدى حساسية الدواء لدى الأطفال

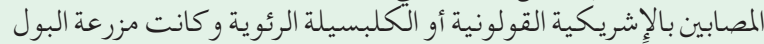

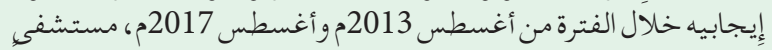

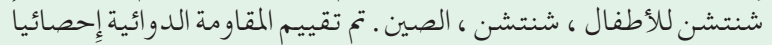

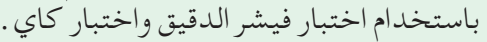

النتائج: أثبتنا 698 حالة من الإشريكية القولونية ، تم تأكيد وجود

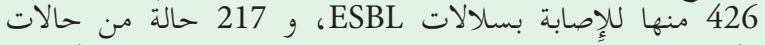

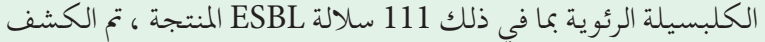

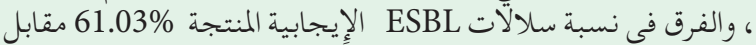

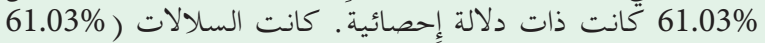

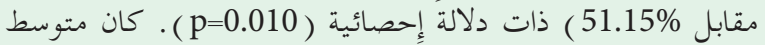

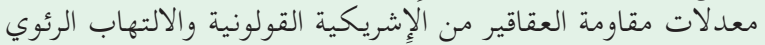

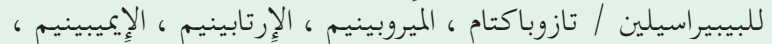

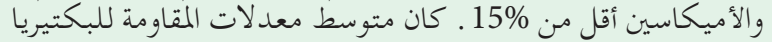

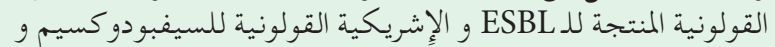

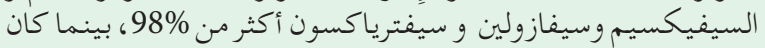

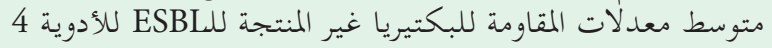

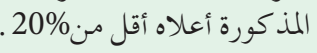

الخلاصة : في جنوب الصين ، كانت نسبة السلالات المنتجة للـ ESBL

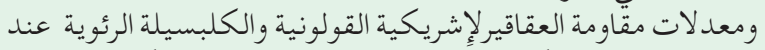

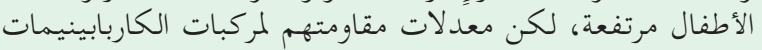

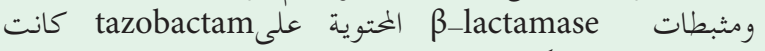

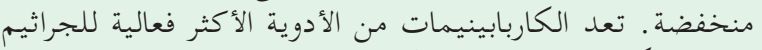
لعلاج البكتيريا المنتجة لـ لـ ESBL.

Objectives: To investigate the drug resistance of extendedspectrum beta-lactamase (ESBL)-producing Escherichia coli (E. coli) and Klebsiella pneumoniae (K. pneumoniae) in children with urinary tract infection (UTI) and to provide the rationale for clinical use of antibiotics.
Methods: This is a retrospective analysis of drug susceptibility in children with $E$. coli or K. pneumoniae-positive urine culture between August 2013 and August 2017, Shenzhen Children's Hospital, Shenzhen, China. Drug resistance was statistically assessed using Fisher exact test and $\chi^{2}$ test.

Results: A total of 698 cases of E. coli, 426 of which were confirmed ESBL-producing strains, and 217 cases of $K$. pneumoniae, including 111 ESBL-producing strains, were detected, and the difference in proportion of positive ESBL-producing strains (61.03\% versus $51.15 \%)$ was statistically significant $(p=0.010)$. The average drug resistance rates of $E$. coli and $K$. pneumoniae to piperacillin/tazobactam, meropenem, ertapenem, imipenem, and amikacin were $<15 \%$. The average resistance rates of ESBL-producing $E$. coli and $K$. pneumoniae to cefpodoxime, cefixime, cefazolin, and ceftriaxone was $>98 \%$, while average resistance rates for non-ESBL-producing bacteria to the above 4 drugs was $<20 \%$.

Conclusion: In southern China, the proportion of ESBL-producing strains and the drug resistance rates of $E$. coli and $K$. pneumoniae in UTI in children was high, but their resistance rates to carbapenems and $\beta$-lactamase inhibitor complexes containing tazobactam were low. Carbapenems are the most effective antibacterial drugs for the treatment of ESBL-producing bacteria.

Saudi Med J 2019; Vol. 40 (11): 1111-1115 doi: 10.15537/smj.2019.11.24547

From the Department of Urology (Keshi, Xiangwei, Rui, Pei), from the Clinical Laboratory (Weiwei), from the Department of Urology (Shoulin, Xiaodong, Hao, Junhai), Shenzhen Children's Hospital,Shenzhen, Guangdong Province, China.

Received 5th May 2019. Accepted 5th September 2019.

Address correspondence and reprint request to: Dr. Wang Xiangwei, Department of Urology, Shenzhen University General Hospital, Shenzhen, Guangdong Province, China. E-mail: winn0324@szu.edu.cn ORCID ID: orcid.org/0000-0001-5484-977X 
U rinary tract infection (UTI) is a common infectious disease in children, and $5.6 \%$ to $10.2 \%$ of children will subsequently develop permanent renal scars, ${ }^{1}$ leading to serious complications such as renal dysplasia, recurrent pyelonephritis, impaired glomerular function, early hypertension, and end-stage renal disease. ${ }^{2}$ Extendedspectrum beta-lactamase (ESBL)-producing Escherichia coli (E. coli) and Klebsiella pneumoniae (K. pneumoniae) are important challenges in the treatment of UTIs in children due to their high incidence and multidrug resistance. There are significant regional differences in the types and detection rates of ESBL-producing bacteria. Studies have shown a gradual increase in the number of ESBL-producing strains isolated from E. coli or $K$. pneumoniae. ${ }^{3}$ Understanding epidemic UTI pathogens and their drug resistance characteristics will improve clinical efficacy and reduce the emergence of drug-resistant strains through empirical drug use. Large numbers of ESBL-producing strains have not been reported in children with UTIs in southern China.

This study intends to analyze the drug resistance of $E$. coli and $K$. pneumoniae, isolated from urine specimens of hospitalized children with UTIs in Shenzhen Children's Hospital, Shenzhen, China thereby providing information on the drug resistance of ESBL-producing bacteria.

Methods. The drug susceptibility results of hospitalized children with UTIs admitted to Shenzhen Children's Hospital between August 2013 and August 2017 were collected. The study included 576 males and 339 females with E. coli- or K. pneumoniae-positive urine cultures with colony number $>10^{5} / \mathrm{ml}$. The ratio of males to females was $1.7: 1$. The age range of the patients was 11 days to 14 years with a median age of 12 months. Patients were excluded if their infections were caused by different pathogens within 2 weeks after anti-infective treatment, or the same pathogen within 4 weeks after anti-infection treatment.

For children who had undergone toilet training, mid-stream urine was collected after cleaning their vulva and urethra. For children with indwelling catheters and non-toilet-trained young children, the catheterization method was applied. Urine specimens were sent for inspection in sterile containers within half an hour of collection. Specimen cultures were performed

Disclosure. This study was funded by the research deputy of the Shenzhen Municipal Health Planning Commission, Shenzhen, China (No. 201507027). according to the requirements of the National Clinical Laboratory Procedures. Strain identification and drug susceptibility tests were carried out by VITEK $^{\oplus} 2$ compact 60 automatic microbial identification and drug sensitivity analyzer (BioMerieux, France). On the basis of the Clinical and Laboratory Standards Institute (CLSI) guidelines, drug resistance, intermediate sensitivity and susceptibility were determined. The minimum inhibitory concentration values were detected by the microdilution method. The standard quality control strains were E. coli ATCC 25922 and K. pneumonia ATCC 700603 (National Center for Clinical Laboratories, Beijing, China). This study has passed the ethical review of the Ethics Committee of Shenzhen Children's Hospital.

Statistical analysis. The data were analyzed by the Statistics Package for Social Sciences for Windows, version 25.0 (IBM Corp, Armonk, NY, USA). The comparison between the data groups was tested by $\chi^{2}$, if the effective value is $<5$, the Fisher exact test was adopted. A $p$ value $<0.05$ was considered statistically significant.

Results. A total of 698 cases of E. coli, 426 of which were ESBL-producing, and 217 cases of $K$. pneumoniae including 111 cases of ESBL-producing strains were detected in this study. The overall prevalence of ESBL-producing strains was $58.69 \%$. The prevalence of ESBL-producing $E$. coli was higher than that of $K$. pneumonia $(61.03 \%$ versus $51.15 \%)$, and the difference in prevalence between them was statistically significant $\left(\chi^{2}=6.664, p=0.010\right)$.

Sensitivity testing of culture strains to antibiotics commonly used in clinical practice showed that the resistance rates of $E$. coli and $K$. pneumoniae to piperacillin/tazobactam, meropenem, ertapenem, imipenem, and amikacin were $<15 \%$.

The resistance rates of ESBL-producing E. coli and $K$. pneumoniae to cefpodoxime, cefaclor, cefazolin, and ceftriaxone were $>98 \%$, whereas the resistance rates of non-ESBL-producing bacteria to the above 4 drugs were $<20 \%$. The difference was statistically significant $(p<0.05)$.

The resistance rates of $E$. coli to nitrofurantoin was $7.61 \%$ and for $K$. pneumoniae was $72.22 \%$, and the difference was statistically significant $\left(\chi^{2}=3.634 \times 10^{2}\right.$, $p=0.000$, Table 1).

Discussion. Since ESBL-producing $K$. pneumoniae was first discovered in the 1980s, it has spread worldwide. There are significant differences in the types and detection rates of ESBL-producing bacteria 
Table 1 - Comparison of drug resistance between Escherichia coli and Klebsiella pneumoniae in extended-spectrum beta-lactamase-producing group and non-extended-spectrum beta-lactamase-producing group (\%).

\begin{tabular}{|c|c|c|c|c|c|c|c|c|c|c|}
\hline \multirow[b]{3}{*}{ Antibacterial drugs } & \multicolumn{5}{|c|}{ Escherichia coli } & \multicolumn{5}{|c|}{ Klebsiella pneumoniae } \\
\hline & \multicolumn{2}{|c|}{ ESBLs (+) } & \multicolumn{2}{|c|}{ ESBLs (-) } & \multirow[b]{2}{*}{$P$-value } & \multicolumn{2}{|c|}{ ESBLs (+) } & \multicolumn{2}{|c|}{ ESBLs (-) } & \multirow[b]{2}{*}{$P$-value } \\
\hline & $\begin{array}{c}\text { Number } \\
\text { of } \\
\text { resistant } \\
\text { strains }\end{array}$ & $\begin{array}{l}\text { Resistance } \\
\text { rate }\end{array}$ & $\begin{array}{l}\text { Number } \\
\text { of } \\
\text { resistant } \\
\text { strains }\end{array}$ & $\begin{array}{l}\text { Resistance } \\
\text { rate }\end{array}$ & & $\begin{array}{l}\text { Number } \\
\text { of } \\
\text { resistant } \\
\text { strains }\end{array}$ & $\begin{array}{l}\text { Resistance } \\
\text { rate }\end{array}$ & $\begin{array}{l}\text { Number } \\
\text { of } \\
\text { resistant } \\
\text { strains }\end{array}$ & $\begin{array}{l}\text { Resistance } \\
\text { rate }\end{array}$ & \\
\hline Ampicillin/sulbactam & 366 & 86.94 & 132 & 55.93 & 0.000 & 109 & 99.09 & 27 & 30.68 & 0.000 \\
\hline Piperacillin/tazobactam & 7 & 1.66 & 3 & 1.27 & 1.000 & 16 & 14.55 & 10 & 11.36 & 0.510 \\
\hline Cefpodoxime & 402 & 100.00 & 7 & 4.05 & 0.000 & 103 & 100.00 & 14 & 16.47 & 0.000 \\
\hline Cefixime & 255 & 100.00 & 3 & 2.07 & 0.000 & 69 & 100.00 & 6 & 11.11 & 0.000 \\
\hline Meropenem & 1 & 0.25 & 1 & 0.44 & 1.000 & 1 & 0.98 & 5 & 6.25 & 0.088 \\
\hline Ertapenem & 5 & 1.19 & 1 & 0.43 & 0.428 & 2 & 1.87 & 7 & 8.54 & 0.738 \\
\hline Aztreonam & 228 & 54.29 & 3 & 1.27 & 0.000 & 81 & 73.64 & 11 & 12.50 & 0.000 \\
\hline Amikacin & 7 & 1.66 & 2 & 0.85 & 0.501 & 2 & 1.82 & 1 & 1.14 & 1.000 \\
\hline Levofloxacin & 157 & 37.20 & 38 & 16.10 & 0.000 & 12 & 10.91 & 9 & 10.23 & 0.877 \\
\hline Nitrofurantoin & 42 & 9.95 & 8 & 3.40 & 0.002 & 82 & 74.55 & 61 & 69.32 & 0.414 \\
\hline Cefazolin & 420 & 99.76 & 27 & 11.44 & 0.000 & 110 & 100.00 & 16 & 18.18 & 0.000 \\
\hline Cefaclor & 406 & 100.00 & 15 & 15.31 & 0.000 & 103 & 100.00 & 13 & 37.14 & 0.000 \\
\hline Ceftazidime & 141 & 33.41 & 5 & 2.12 & 0.000 & 69 & 62.73 & 14 & 15.91 & 0.000 \\
\hline Ceftriaxone & 420 & 99.53 & 13 & 5.51 & 0.000 & 108 & 98.18 & 15 & 17.05 & 0.000 \\
\hline Cefepime & 138 & 32.70 & 2 & 0.85 & 0.000 & 55 & 50.00 & 10 & 11.49 & 0.000 \\
\hline Imipenem & 1 & 0.24 & 1 & 0.42 & 1.000 & 6 & 5.45 & 10 & 11.36 & 0.130 \\
\hline Cefotetan & 6 & 1.42 & 2 & 0.85 & 0.718 & 5 & 4.55 & 12 & 13.64 & 0.023 \\
\hline Tobramycin & 179 & 42.42 & 73 & 31.06 & 0.004 & 42 & 38.18 & 10 & 11.36 & 0.000 \\
\hline Gentamicin & 174 & 41.23 & 75 & 32.05 & 0.020 & 38 & 34.55 & 9 & 10.23 & 0.000 \\
\hline Ciprofloxacin & 175 & 41.47 & 38 & 16.10 & 0.000 & 21 & 19.09 & 10 & 11.49 & 0.146 \\
\hline Sulfamethoxazole & 272 & 64.45 & 102 & 43.40 & 0.000 & 61 & 55.45 & 18 & 20.45 & 0.000 \\
\hline
\end{tabular}

in various countries and regions. Escherichia coli is the main pathogen causing UTI; E. coli can invade bladder epithelial cells, escape into the cytoplasm of host cells and form intracellular bacterial communities, allowing bacterial replication and avoiding host immune responses. ${ }^{4}$ Escherichia coli and K. pneumoniae are the most common ESBL-producing bacteria; indwelling catheterization, history of recurrent urinary tract infection and use of antibiotics within 3 months are risk factors for ESBL production. ${ }^{5}$ The detection rates are higher in Latin America, Asia, and the Middle East but lower in the South Pacific, Europe, and North America, ${ }^{6,7}$ considering the abuse and non-standard use of antibiotics in developing countries. It has been reported that from 2000 to 2009 , the detection rate of ESBL-producing E.coli increased from 3.3\% to $8 \%$ and that of $K$. pneumoniae increased from $9.1 \%$ to $18.6 \% .^{8}$ The overall rate of UTIs, caused by ESBL-producing bacteria, in our hospital was $58.69 \%$, and the proportions positive for ESBLs-producing was $61.03 \% \mathrm{E}$. coli and $51.15 \% \mathrm{~K}$. pneumoniae. These data indicate that the region has a high detection rate of ESBL-producing bacteria. Extended-spectrum beta-lactamases are plasmid-mediated, widely active $\beta$-lactamases that hydrolyze penicillins and cephalosporins by cleavage of the amide bond of the $\beta$-lactam ring to inactivate $\beta$-lactam antibiotics; therefore, inferring resistance to first to 3rd generation cephalosporins and aztreonan antibiotics. ${ }^{9}$ At the same time, these plasmids usually carry additional resistance genes to other drugs, such as aminoglycoside, sulfonamides, and quinolones, to make the bacteria multidrug-resistant, ${ }^{10}$ which often leads to serious infections. By understanding the epidemiological and drug resistance characteristics of ESBL-producing bacteria, antibiotics can be used in a targeted manner to avoid inappropriate antibacterial treatment and the increase of resistant strains producing ESBLs.

In this study, ESBL-producing E. coli and $K$. pneumoniae were widely resistant to cephalosporins. In addition to cefotetan, resistance to cefepime, a 4th generation cephalosporin, was evident in $32.7 \%$ of cases. Thus, we recommend that unless there is a clear indication of drug susceptibility, cephalosporin should be cautiously used for treatment of UTIs in children with ESBL-producing bacteria. The activity of ESBL- 
producing bacteria can be inhibited by $\beta$-lactamase inhibitors such as sulbactam, clavulanic acid, and tazobactam. The currently recommended antibacterial agents for ESBL-producing bacteria are combination preparations of carbapenems and $\beta$-lactamase inhibitors. It is also possible to use aminoglycosides and fluoroquinolones in combination with the above drugs based on drug susceptibility results and conditions. Carbapenems are considered first-line treatment for ESBL infections. ${ }^{11}$ Extended-spectrum betalactamase-producing bacteria are sensitive to different combination preparations, such as cefoperazone/ sulbactam and piperacillin/tazobactam, but resistant to amoxicillin/clavulanic acid and ampicillin/sulbactam. This is consistent with the results of our study, which showed low resistance rates of ESBL-producing (1.66\% E. coli and (14.55\%) K. pneumoniae) to piperacillin/ tazobactam and high resistance rates to ampicillin/ sulbactam (86.94\% E. coli and $99.09 \%$ K. pneumoniae). A combination of piperacillin/tazobactam is preferred for mild to moderate infections caused by ESBL-producing bacteria, whereas carbapenems may be selected for severe infections. While other antibacterial agents have poor efficacy, carbapenem antibiotics act quickly and stably and can resist the hydrolysis of ESBLs, as supported by sufficient clinical data. Therefore, carbapenems are currently the most effective antibacterial agents for the treatment of ESBL-producing bacteria. ${ }^{11-13}$ The resistance rate to meropenem, ertapenem, and imipenem in this group was $0.24 \%-5.45 \%$, which confirmed the high sensitivity of carbapenem antibiotics to ESBLs-producing bacteria. It is worth noting that 3 carbapenem antibiotics that produce ESBLs from $E$. coli and $K$. pneumoniae in in vitro susceptibility tests have been found in the drug-resistant strains. Therefore, it is necessary to strictly control the indications for drug use in clinical treatment. Further increase of drug-resistant strains can be avoided by adequate medium dose, appropriate course of treatment, and alternating use of antibacterial drugs.

Although ESBL-producing bacteria carry aminoglycoside-resistant genes, our study found that ESBL-producing bacteria have a resistance rate of $<2 \%$ to amikacin, which is significantly lower than that of gentamicin and tobramycin. However, these drugs have ear and kidney toxicity in children and need to be used with caution. Therefore, they are only used as a combination therapy for patients with severe infections caused by ESBL-producing bacteria. Ciprofloxacin and levofloxacin affect cartilage development in infants and young children, and their sensitivity rate in our study has no obvious advantage over aminoglycoside and $\beta$-lactamase inhibitor complex preparations, which is consistent with the resistance of fluoroquinolones to gram-negative bacilli causing UTIs found by Hoban et al. ${ }^{14}$ Hence, we do not recommend the use of fluoroquinolone antibiotics for the treatment of UTIs in children.

Some studies have shown that nitrofurantoin has better kidney metabolism and is recommended for the treatment of complex UTIs. ${ }^{15}$ However, there are also studies suggesting that nitrofurantoin is only used as a preventive drug and not for the treatment of complicated UTIs. ${ }^{16}$ The American Urological Association 2010 guidelines consider that there is no theoretical basis for continuous prophylactic use of antibiotics. It is recommended that infants with only vesicoureteral refluxand those with bladder and bowel dysfunction have prophylactic antibiotics. In our study, the resistance rates of E. coli and $K$. pneumoniae to nitrofurantoin were significantly different $(7.61 \%$ versus $72.22 \%$ ). We recommend nitrofurantoin as a preventive medication for the treatment of UTIs caused by $E$. coli and recurrent UTIs. The gastrointestinal side effects of nitrofurantoin in children need to be considered while using the drug.

Study limitation. It is only a single-center study, and the time span is not long enough to reflect the trend of ESBL- producing bacteria; multi-center, longer-term research would be more reflective of the real situation. It is necessary to strictly control the indication for medication and adjust the antibacterial drugs over time according to the results of drug susceptibility tests. The use of antibiotics in sufficient amounts and over sufficient courses of treatment can reduce the production of resistant bacteria producing ESBLs and improve the clinical treatment effect.

This study will advise clinicians in the region on the clinical use of drugs, it will simultaneously inform other regions on the situation of ESBL-producing bacteria in South China.

In conclusion, the proportion of UTIs in children caused by ESBL-producing bacteria is high in South China, and the drug resistance is serious. For the mild to moderate UTIs caused by ESBL-producing $E$. coli and $K$. pneumoniae, the $\beta$-lactamase inhibitor complex containing tazobactam should be preferred. Carbapenems can be selected for patients with severe or poor efficacy. Nitrofurantoin can be used to prevent UTIs caused by E. coli. 
Acknowledgment. The authors gratefully acknowledge Shenzhen Municipal Health Planning Commission, Shenzhen, China for funding this study.

\section{References}

1. Keren R, Shaikh N, Pohl H, Gravens-Mueller L, Ivanova A, Zaoutis $\mathrm{L}$, et al. Risk factors for recurrent urinary tract infection and renal scarring. Pediatrics 2015; 136: 13-21.

2. Stein R, Dogan HS, Hoebeke P, Kočvara R, Nijman RJM, Radmayr C, et al. Urinary tract infections in children: EAU/ ESPU guidelines. Eur Urol 2015; 67: 546-558.

3. Castanheira M, Mills JC, Costello SE, Jones RN, Sader HS. Ceftazidime-avibactam activity tested against Enterobacteriaceae isolates from US hospitals (2011 to 2013) and characterization of $\beta$-lactamase-producing strains. Antimicrob Agents Chemother 2015; 59: 3509-3517.

4. Conover MS, Hadjifrangiskou M, Palermo JJ, Hibbing ME, Dodson KW, Hultgren SJ. Metabolic requirements of Escherichia coli in intracellular bacterial communities during urinary tract infection pathogenesis. MBio 2016; 7: e00104-e00116.

5. Goyal D, Dean N, Neill S, Jones P, Dascomb K. Risk factors for community-acquired extended-spectrum beta-lactamaseproducing Enterobacteriaceae infections-a retrospective study of symptomatic urinary tract infections. Open Forum Infect Dis 2019; 6: 357.

6. Morrissey I, Hackel M, Badal R, Bouchillon S, Hawser S, Biedenbach D. A review of ten years of the study for monitoring antimicrobial resistance trends (SMART) from 2002 to 2011. Pharmaceuticals 2013; 6: 1335-1346.

7. Vega S, Dowzicky MJ. Antimicrobial susceptibility among Gram-positive and Gram-negative organisms collected from the Latin American region between 2004 and 2015 as part of the tigecycline evaluation and surveillance trial. Ann Clin Microbiol Antimicrob 2017; 16: 50.
8. Zilberberg MD, Shorr AF. Secular trends in Gram-negative resistance among urinary tract infection hospitalizations in the United States, 2000-2009. Infect Control Hosp Epidemiol 2013; 34: 940-946.

9. Hsu AJ, Tamma PD. Treatment of multidrug-resistant Gramnegative infections in children. Clin Infect Dis 2014; 58 : 1439-1448.

10. Gupta K, Bhadelia N. Management of urinary tract infections from multidrug-resistant organisms. Infect Dis Clin North Am 2014; 28: 49-59.

11. Delbet JD, Lorrot M, Ulinski T. An update on new antibiotic prophylaxis and treatment for urinary tract infections in children. Expert Opin Pharmacother 2017; 18: 1619-1625.

12. Bader MS, Loeb M, Brooks AA. An update on the management of urinary tract infections in the era of antimicrobial resistance. Postgrad Med 2017; 129: 242-258.

13. Lob SH, Hackel MA, Hoban DJ, Young K, Motyl MR, Sahm DF, et al. Activity of Ertapenem against Enterobacteriaceae in seven global regions-SMART 2012-2016. Eur J Clin Microbiol Infect Dis 2018; 37: 1481-1489.

14. Hoban DJ, Lascols C, Nicolle LE, Badal R, Bouchillon S, Hackel $\mathrm{M}$, et al. Antimicrobial susceptibility of Enterobacteriaceae, including molecular characterization of extended - spectrum beta - lactamase - producing species, in urinary tract isolates from hospitalized patients in North America and Europe: results from the SMART study 2009-2010. Diagn Microbiol Infect Dis 2012; 74: 62-67.

15. Munoz-Davila MJ. Role of old antibiotics in the era of antibiotic resistance. highlighted nitrofurantoin for the treatment of lower urinary tract infections. Antibiotics (Basel) 2014; 10: 39-48.

16. Vélez EC, Serna-Higuita LM, Serrano AK, Ochoa-García C, Rojas Rosas L, María Bedoya A, et al. Resistance profile of pathogens causing urinary tract infection in the pediatric population, and antibiotic treatment response, at a University Hospital 2010-2011. Colomb Med (Cali) 2014; 45: 39-44. 